\title{
Mobile drone LiDAR structural data collection and analysis
}

\author{
CNC Baylis BHP, Australia \\ DR Kewe BHP, Australia \\ EW Jones BHP, Australia
}

\begin{abstract}
The BHP Olympic Dam underground mine site has experienced ongoing issues with overbreak within its stopes in the recently-developed Southern Mining Area. The rock mass is poorly characterised and there are limited data on key structures influencing the ultimate shape of stopes and the extent of overbreak. The introduction of the Emesent Hovermap mobile drone LiDAR system has provided more extensive, accurate scan coverage of existing stopes, some which were previously inaccessible via earlier scanning methods. This new approach provides a much higher resolution scan than does the industry standard Cavity Monitoring System. With these data, a new methodology of digitally geo-mapping exposed structures within the stopes has been developed to create a new dataset. This is now used for further mine planning through comparing, validating, and appending to modelled geological structural features. This paper discusses the process of acquiring and preparing drone-based LiDAR data for geomechanics and explores the spatial and structural relationship between drone data and window mapping for implementation into Olympic Dam's future stope designs. The back-analysis of stope failures through the LiDAR scan structural mapping has provided a great deal of insight into structural characterisation of underground areas and can assist in planning future stopes. It has not only provided more detailed information on the previously identified large persistent structures but has also identified large multi-stope spanning structures which were previously unrecognised. Evidence of broader zones of weakness, more extensive than previously appreciated, were also uncovered through small-scale structural persistence through mine areas.
\end{abstract}

Keywords: LiDAR, structure, underground, stope, drone, geo-mapping

\section{Introduction}

BHP Group Ltd has operated Olympic Dam (OD) since 2005 following its acquisition of Western Mining Corporation. It is located approximately $560 \mathrm{~km}$ north-northwest of Adelaide, South Australia (Figure 1). The large polymetallic orebody was discovered in 1975 as a uranium-bearing 'iron oxide copper gold' type deposit. Production commenced in 1988 utilising the sublevel open stope mining method. OD is one of the largest such mines in the world; it currently produces $171,600 \mathrm{t}$ of copper cathode, 146,000 oz of gold, 984,000 oz of silver and 3,678 t of uranium concentrate (BHP 2020).

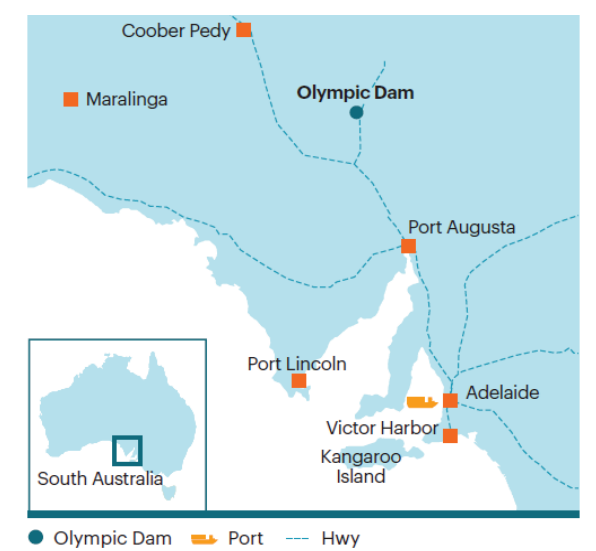

Figure 1 Olympic Dam is located in central South Australia, approximately 560 km north-northwest of Adelaide 
Stopes at OD are typically aligned on a $30 \times 30 \mathrm{~m}$ or $25 \times 25 \mathrm{~m}$ grid footprint. Heights range from single lift $30 \mathrm{~m}$ high stopes, to multi-lift stopes exceeding $200 \mathrm{~m}$ in height. Stopes are typically blasted in two or more blast packets (Figure 2) and at minimum, each stope is accessible from the drawpoint and crown levels. Larger stopes may also have one or more mid-level accesses.
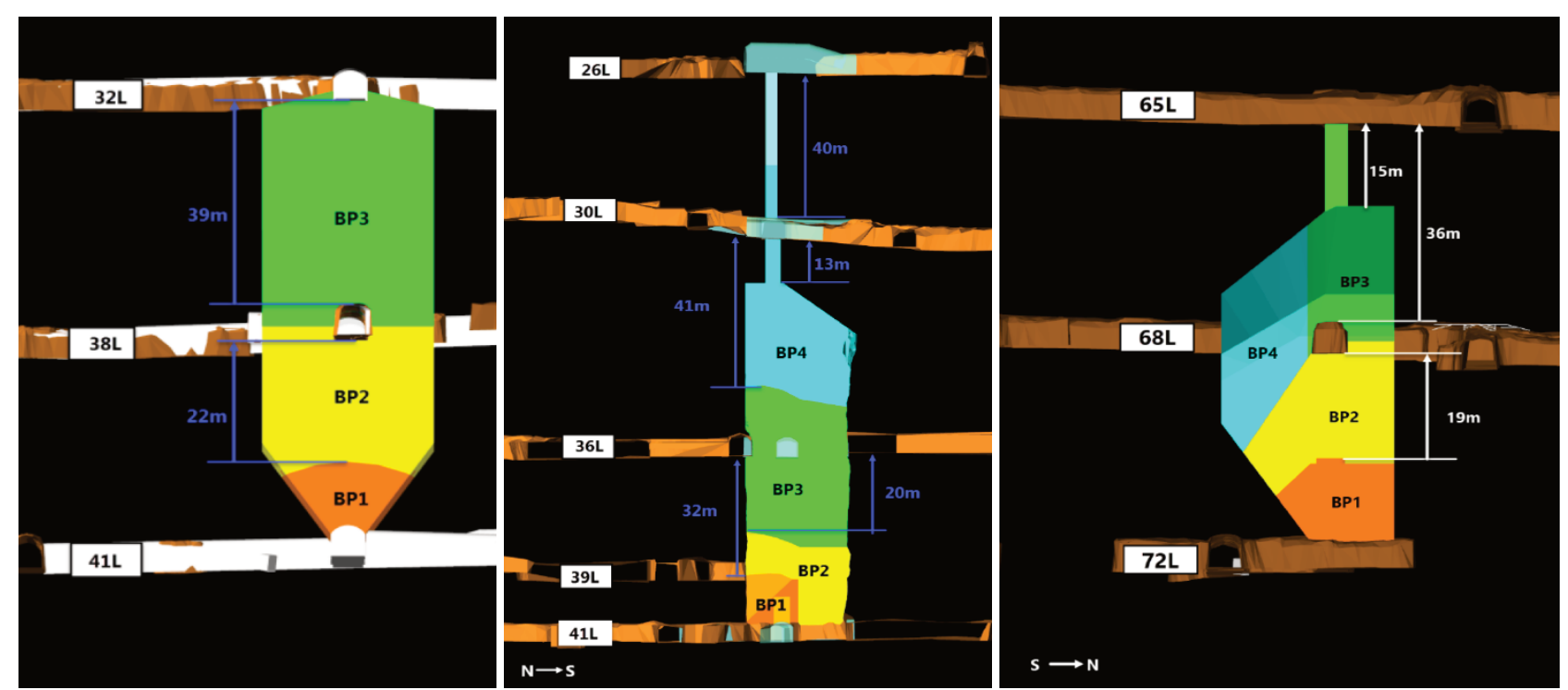

Figure 2 Underground blast packet series mining method-sublevel open stope

The underground mining operations continue to $~ 900 \mathrm{~m}$ below surface and extend approximately $6 \mathrm{~km}$ along strike from north-west to south-east; and $3 \mathrm{~km}$ north-east to south-west. The northern part of the mine, known as the Northern Mining Area (NMA), has been continuously mined over the last 30 years and its mineable resources are now diminishing. The focus has therefore turned to developing new mining blocks like the deeper areas of the NMA, such as the Deep Greens and Deep Purples, as well as accessing new lodes within the Southern Mining Area (SMA); the Teals, Tangerines and Emeralds. Thirty years of mining the NMA has provided a great deal of experience and a detailed understanding of the expected rock mass conditions, with well-rehearsed processes for the design and extraction of these stopes. Occasionally, isolated pockets of poorer rock mass conditions were encountered in the NMA, resulting in increased challenges during planning and extraction. In general, the rock mass was well understood and stopes performed as planned. By contrast, the SMA has proven more challenging as it has presented a range of new lithological units, a higher degree of alteration throughout the rock mass, and a greater influence of regional, deposit and lode-scale structures. The result of this geological variability is a higher degree of geotechnical uncertainty, variance in rock mass parameters and spatial variability. This contrast in rock mass characteristic means that planning and extraction techniques previously appropriate for the NMA are being revised based upon the anticipated geotechnical parameters in the SMA.

The mine layout is shown in Figure 3, outlining the major mining blocks, along with the major regional-scale Mashers Fault, and newly encountered weak volcaniclastic sediments (KASH) and highly altered Northern and Southern Structural Gouge Zone (NSGZ and SSGZ, respectively) rock units. 


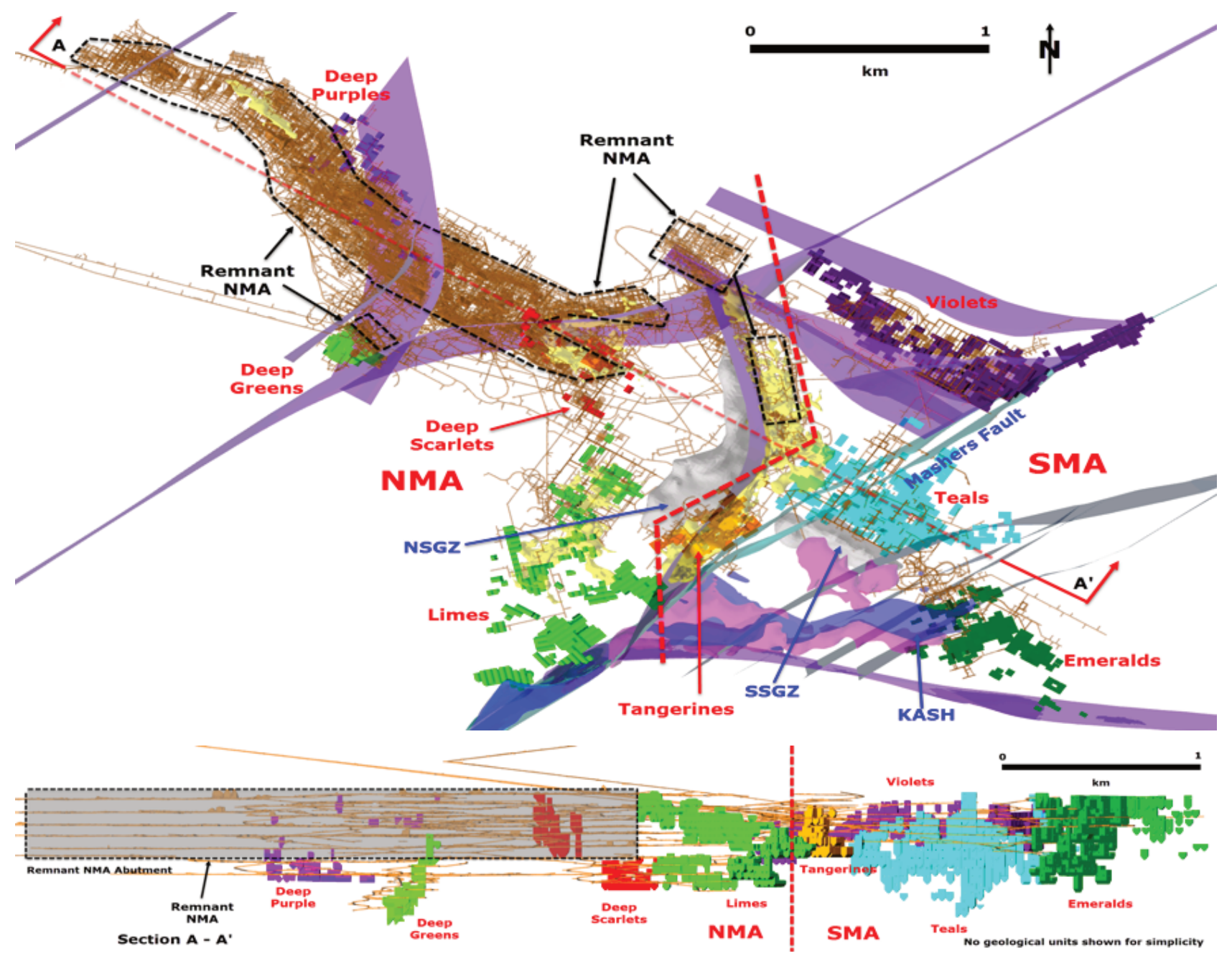

Figure 3 Overview of the Olympic Dam mine layout showing the new mining areas in the Southern Mining Area (SMA) and Northern Mining Area (NMA) extensions. Major geological units and features are also shown (Jones et al. 2020)

As production has advanced into the new mining areas, there has been a focus on new ways to rapidly acquire rock mass data. One of the adopted methods has used LiDAR point clouds produced from semi-autonomous drones flown into stope voids. Since February 2019, OD's survey and production departments have adopted the Emesent Hovermap drone-base mobile LiDAR scanner, scanning stopes as a replacement to conventional Cavity Monitoring System (CMS) methods. Soon after, the geomechanics department started repurposing the data, researching methods for characterising the rock mass, reviewing in detail the methods and timing of failure within stopes, and revaluating design assumptions through back-analysis.

\section{Technology}

The Australian Commonwealth Scientific and Industrial Research Organisation (CSIRO) developed the early prototypes of the Hovermap drone-based mobile LiDAR system. The system involved a combination of industrial robotics and 3D simultaneous localisation and mapping algorithms (Jones et al. 2020).

The Hovermap LiDAR scanner consists of a multi-channel Velodyne LiDAR sensor (VLP-16), an inertial measurement unit (IMU), and an onboard computer. The VLP-16 sensor component is a static scanner with a $360^{\circ}$ horizontal view with a $+/-15^{\circ}$ vertical field of view. In ideal conditions, the scanner produces 300,000 points per second, to a range of $100 \mathrm{~m}$ and at accuracy at range of $+/-30 \mathrm{~mm}$. To produce a spherical field of view, the VLP-16 sensor is mechanically rotated $360^{\circ}$, perpendicular to the sensor's horizontal field of view. 
At OD, the Hovermap scanner is coupled to a DJI Enterprise M210 Drone (Figure 4). The real-time LiDAR results integrate into the drone flight controller enabling autonomous position hold, collision avoidance and route setting in GPS-denied and dark environments.

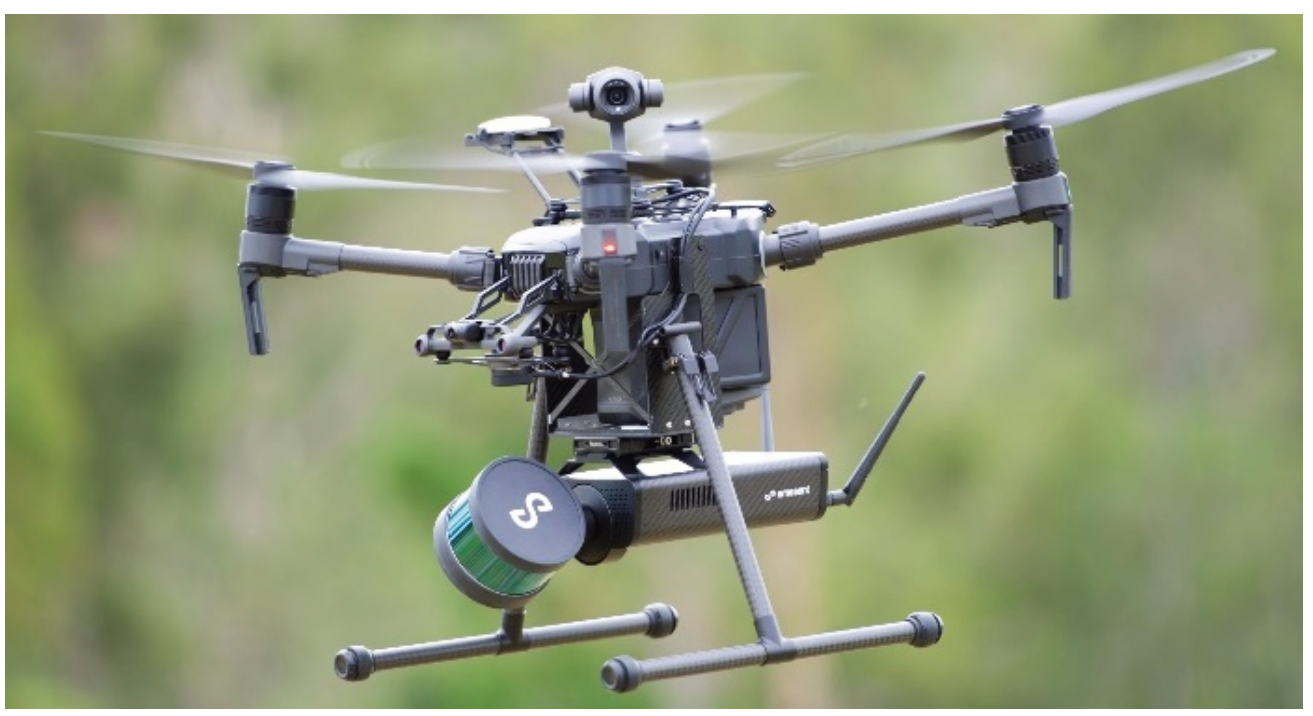

Figure 4 LiDAR system mounted onto a DJI Enterprise M210 Drone (Jones et al. 2020)

\section{Deployment of Hovermap at Olympic Dam}

Emesent initially delivered the Hovermap system to OD in February 2019. Throughout the year, underground operators were trained and upskilled in the piloting and use of the Hovermap system. More details on the implementation may be found in Woolmer et al. (2021).

The process of collecting in-stope LiDAR scans has been integrated as standard practice in the underground 8-day operational scheduling workflow (Figure 5). Engineers, surveyors, or geologists initially identify scan locations and submit a request defining the target and purpose of the scan. These requests are integrated into the 8-day operational schedule. Adequate planning ensures that all scans are appropriately resourced by hardware, pilot availability, and site preparation. Scan priorities vary and some may take precedence over scheduled production. The setup and conduct of the scan itself occupies only 10 minutes. Each day, many scans per drone can be scheduled. They are quick, accurate, and they reduce the exposure of people to the hazards of proximity to the stope void.
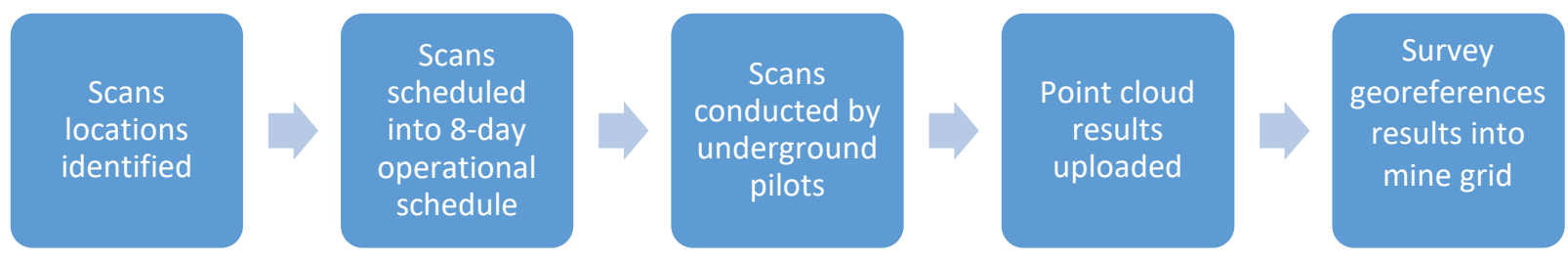

\section{Figure 5 Deployment of Hovermap at Olympic Dam Workflow}

Stope scans each generate about $1 \mathrm{~GB}$ of data, uploaded nightly in the open-source .laz or .las file format. This format can be visualised in several software packages. The raw data is post-processed by the drone operators and two files are provided for use on the surface; the full raw point cloud and a subsampled low-resolution point cloud. This reduces processing time on the surface and ensures hardware rarely comes above ground. Initial result files are not georeferenced in the mine's grid system; this step is completed by the survey department using solids created from low-resolution scans. The georeferenced shape is then uploaded to the site's mine CAD software, DeswikCAD (Deswik 2019). The solid shapes are then used downstream for engineering, void management, and reconciliation. 


\section{Data preparation}

Conventional mining engineering software typically uses a combination of strings, triangulations and wireframed meshes for the visualisation of mining geometry. This rendering method enables sparse data to be visualised as a solid continuous shape using a relatively low number of measured data points as the mesh vertices. Conventionally, the application of the data was to provide guidance on the location and shape of excavations.

The software used in the following research is the open-source package CloudCompare (CloudCompare 2020). This software contains various built-in tools enabling a qualitative and quantitative analysis of the point clouds to be undertaken. There are advantages with working with point clouds over meshes. Each LiDAR point contains information beyond just position $(X, Y, Z)$ in space such as:

- Time.

- Range.

- Reflectance intensity.

- Channel number (when the sensor is using multiple LiDARs concurrently).

- Return number (when using a dual-return LiDAR).

As the purpose for stope scans now includes the recognition of structure at scales of metres to tens-of-metres (similar to that of drive mapping), there is a requirement to have much higher density point cloud. Furthermore, there is a distinct advantage in having a regular point density throughout the entirety of the scan. The use of a mobile-scanning platform enables access to more of the stope and to scan it from multiple angles, reducing the effect of shadowing found from a single stationary scan.

Prior to any analysis, the point clouds are georeferenced to the mine's grid system. As such, all data extracted from the point clouds can be uploaded into the mine's database with Deswik.MDM, the software used by planning and technical departments at OD.

The LiDAR hardware creates a raw data point cloud with the aforementioned attributes. Prior to any analysis, the raw dataset undergoes a QA/QC process. This process checks for any scan errors, identifies gaps or 'shadows', and identifies limitations in the dataset. Issues that have been identified through this process have included the misalignment of one of the LiDAR channels and failed mutual alignment of the points 'sent' with those subsequently 'received'. Jones et al. (2019) discusses other aspects of the QA/QC process.

The initial cloud point density far exceeds that required for structural analysis. The full dataset is therefore subsampled at $\sim 250 \mathrm{~mm}$ spaced data points, $\sim 25$ points per $\mathrm{m}^{2}$, referred to as a low-resolution point cloud, which is used for wireframing and the volumetric and over/underbreak analysis. This low-resolution point cloud can follow the conventional workflow previously established for wireframing and visualisation as for CMS scans. The low-resolution point cloud typically has fewer datapoints than a CMS scan; a saving made possible because the points are chosen to be equally spaced where coverage permits.

Through the proof-of-concept stages of this research, it was found that higher resolution data at $25 \mathrm{~mm}$ spacing, 1,681 points per $\mathrm{m}^{2}$, provided a greater ability for picking features. The improvements in a high-resolution scan compared to a conventional CMS is discussed in Vallejos (2019, p. 54). A full stope scan is typically in the range of 40-120 million data points, depending on the size of the stope and the scan time.

A comparison in the datasets between a CMS, low-resolution, high-resolution and raw point cloud are shown in Figure 6. 


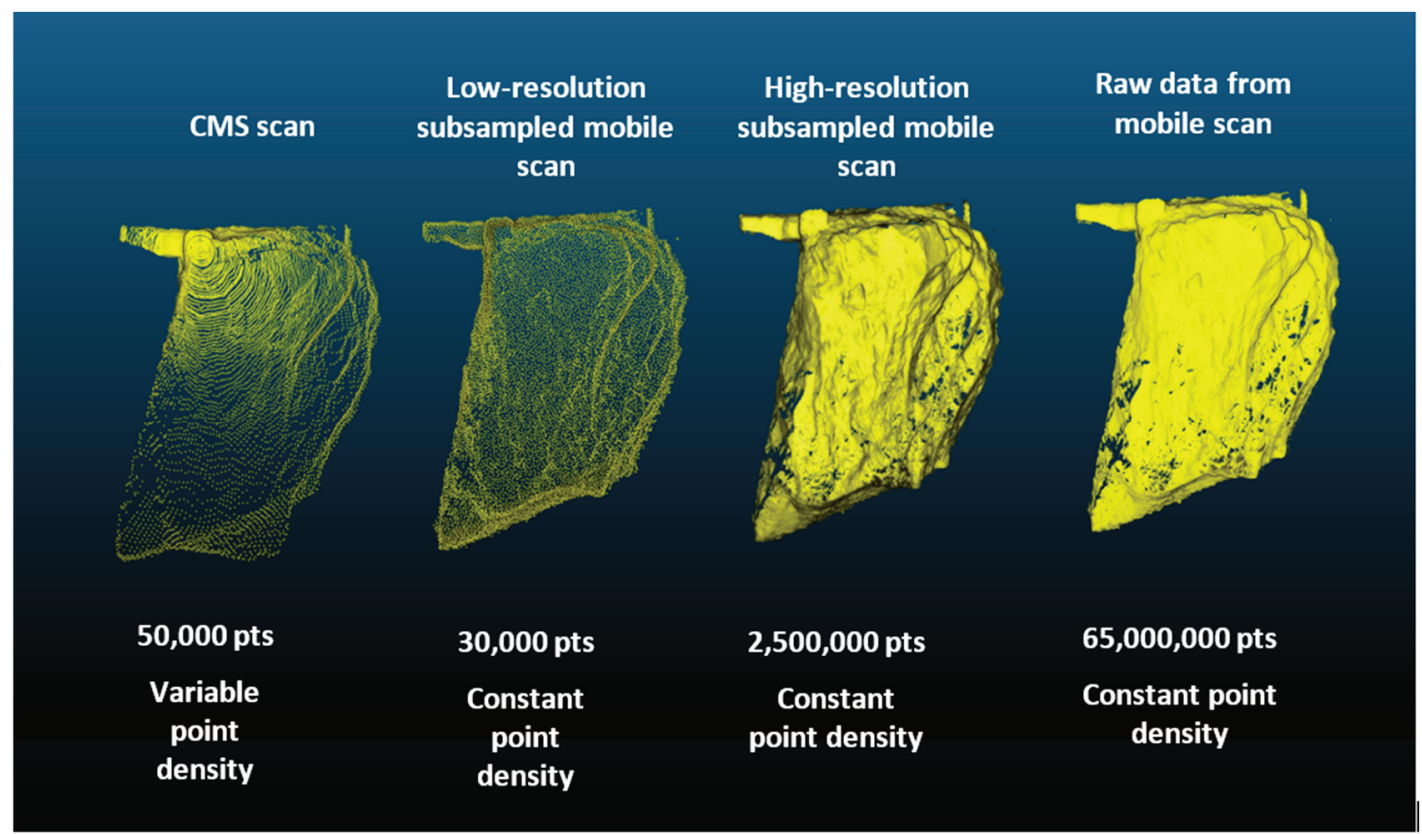

Figure 6 Comparison of various scanning methods and the corresponding number of points. From left to right: CMS scan, low-resolution scan used for wireframing, high resolution scan used for structural picking, raw data from scan

LiDAR scans are completed at regular intervals through the stope's extraction. This allows better understanding of stope performance and the identification of emerging issues. The same geo-referencing process is repeated for each scan and when superimposed, highlights the progression of the extraction and shape over time (Figure 7).

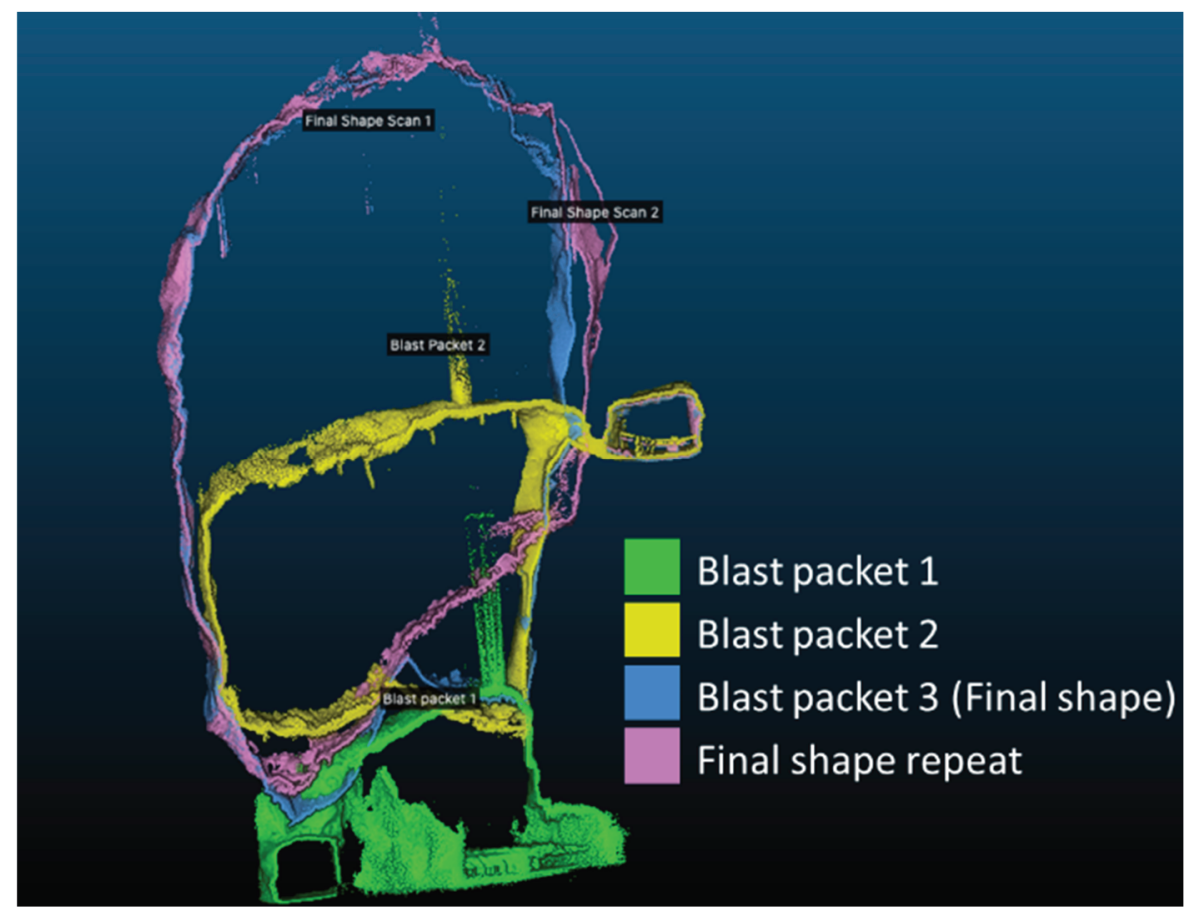

Figure 7 Multiple LiDAR scans of the same stope over time

Once all existing scans are georeferenced, all other relevant geological and geotechnical data, such as faults, joints, shear zones and dykes are added to the model in CloudCompare. Analysis can then be undertaken with all the data to guide extraction of new information from the stope. 


\section{$5 \quad$ Geotechnical analysis}

Vallejos (2019), Jones et al. (2020), and Woolmer et al. (2021) document geotechnical analysis from point clouds. In this paper, we are going to focus on extracting structure from various stopes in a method that is being trialled at OD. The two aspects of this, which will be discussed, are structural picking and implementation into mine databases on site.

\subsection{Structure picking}

Analysis of geology and geomechanics of the stope scan commences by identifying visible planar facets within the LiDAR stope void scans. These may result from any one of several geological features: a joint, fault, shear zone, dyke or rock unit contact. The type of planar discontinuity can be estimated through the supplementation of existing information in the stope, such as known faults with similar position and orientation, and by the nature of the feature; a consistent set of planes with similar dip and dip direction is often characterised as a set of joints. Picking these facets in CloudCompare is done with the 'compass' tool for measuring outcrops. During analysis, the discontinuities identified are based on large obvious structures seen in the scan (Figure 8). A limitation of this tool, however, is the ability to extract only planar facets; discontinuities exhibiting curvature are not well mapped.

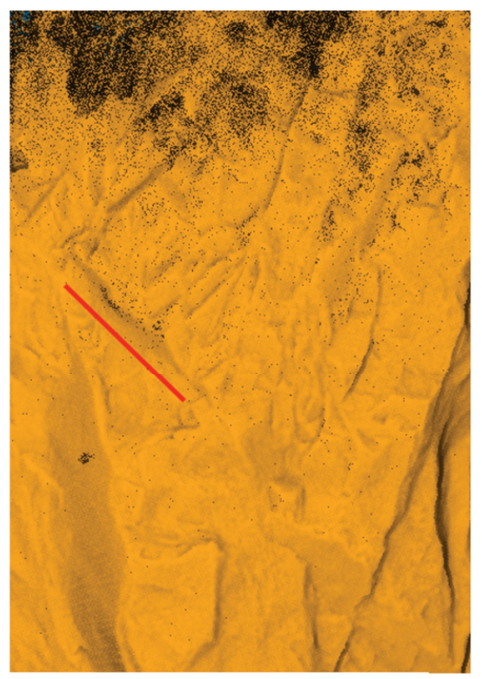

(a)

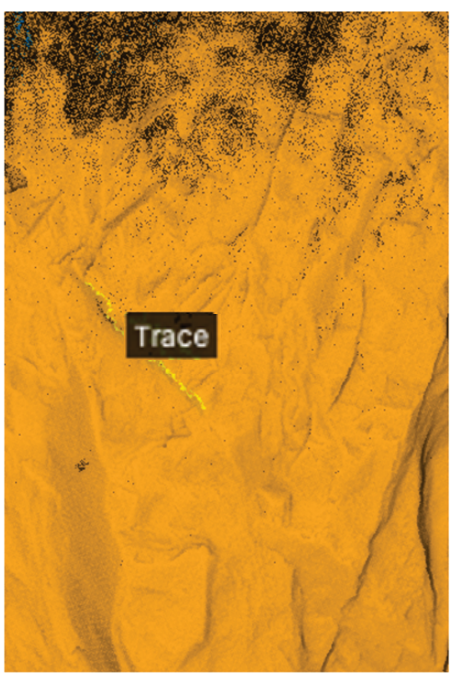

(b)

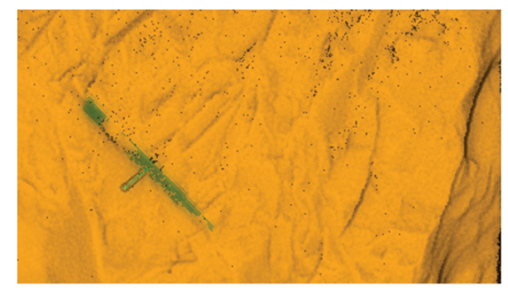

(c)

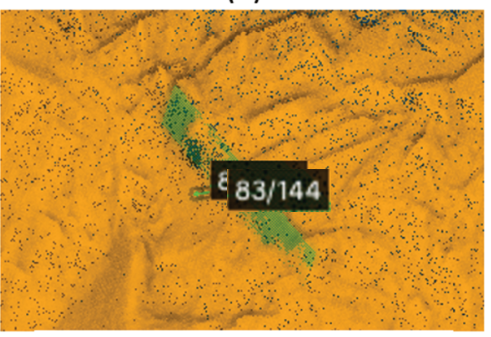

(d)

Figure 8 CloudCompare discontinuity picking tool. (a) Viable planar discontinuity; (b) Traced using 'trace tool' in CloudCompare; (c) Planar facet fitted to the plane; (d) Different perspective of the facet, with a dip and dip direction

Large (stope-scale) discontinuities are identified first. Due to their size, their shape is deduced with a high degree of confidence. As the stope enlarges with each additional blast packet, the corresponding scans produced show the progressive failure along these discontinuities. This improves confidence in modelling as with this method, discontinuities are exposed in the stope over long continuous intervals. This is a major advantage over other underground mapping methods, such as drilling or window mapping, where evidence of the discontinuity persistence can be hard to determine. These large discontinuities can then be wireframed and uploaded to on-site mine databases. Once these stope-scale discontinuities are mapped, smaller scale discontinuities are identified, first in the crown, then in the walls. These discontinuities have a persistence of not less than the width of a drive $(\sim 5 \mathrm{~m})$. Once completed, the discontinuities modelled give an indication of the 3D fracture network of the volume of rock. These discontinuity spatial data are exported in a CSV format and can be used in analysis. 


\subsection{Implementation of the data}

The large underground discontinuities identified through stope-scale discontinuities can be previously known or previously unsuspected. The geology team incorporate both this underground information and independent data from diamond drilling into a 3D structural model. The geotechnical and mine design engineers rely on the 3D modelled discontinuities to forecast underground stability and to set design parameters. The integration of LiDAR scans into the acquisition of underground discontinuity models is therefore a possible way of improving this process. LiDAR has the advantage of being highly accurate, fast, and simple to set up. An example of this is displayed in Figure 9.

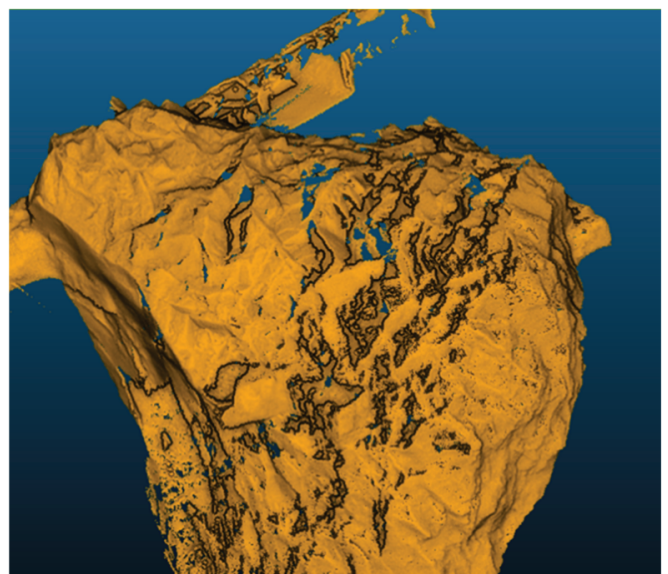

(a)

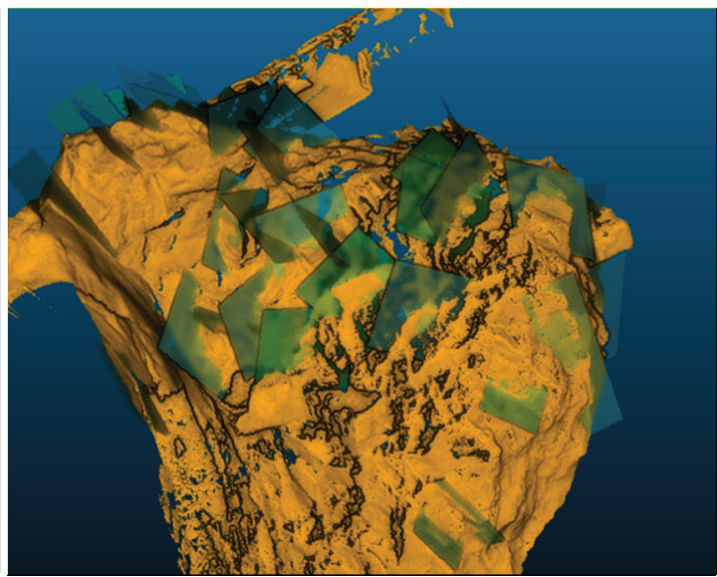

(b)

Figure 9 (a) High resolution LiDAR scan of stope void crown; (b) Mapped discontinuities planes

Small discontinuities are used to gain a better understanding of the rock mass in terms of geotechnical behaviour. Once the stope has been analysed and discontinuities extracted (Figure 9), the dip and dip direction data are exported and used in Dips (Roscience 2020) to analyse joint sets, failure mechanisms and rock mass characteristics (see Figure 10). The results from Figure 10 show the comparison between underground mapped joint sets and LiDAR extracted discontinuity. The variation of the minor discontinuities sets is relatively higher than window mapping data. However, the major joint sets between the two stereonets show some correlation. The variation between window mapping and LiDAR is a focus of current research.

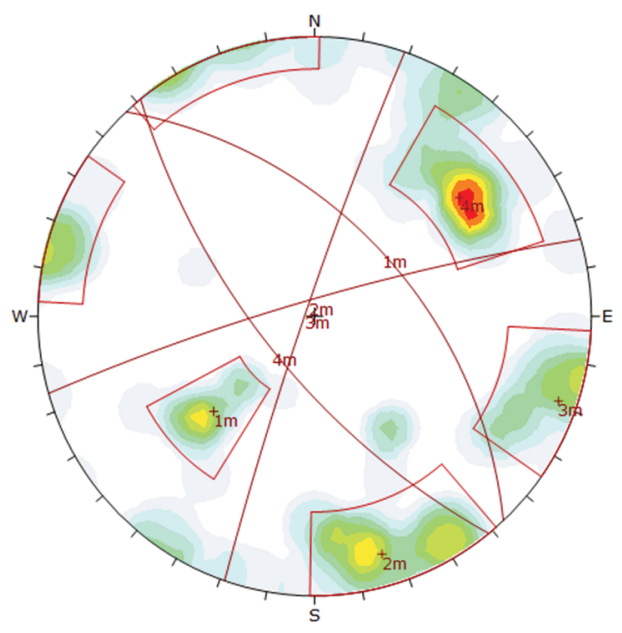

(a)

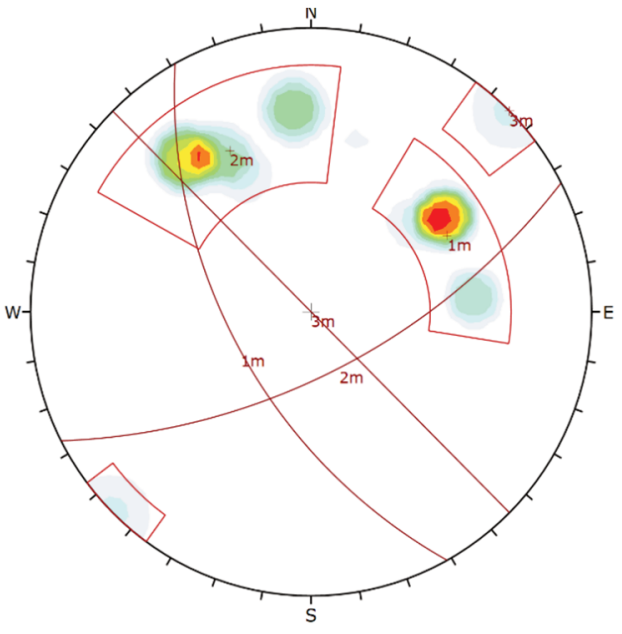

(b)

Figure 10 Stope from Figure 9 stereonets. (a) Stereonet of window mapping data used in stope stability assessments; (b) Stereonet of LiDAR scan extracted discontinuities 
Performing these analyses and data collection across entire mining blocks, such as the tangerines in Figure 11, allows for better characterisation of the rock mass and provides additional data for future stopes in the block. Large-scale discontinuities can be traced through multiple stopes and improve the geology team's confidence in the position and character of the feature. Small-scale structures, such as joints, can help characterise the rock mass, and map its variation across the mine block. This combined information from the LiDAR data can facilitate more informed decision making when designing future stopes adjacent to past stopes.

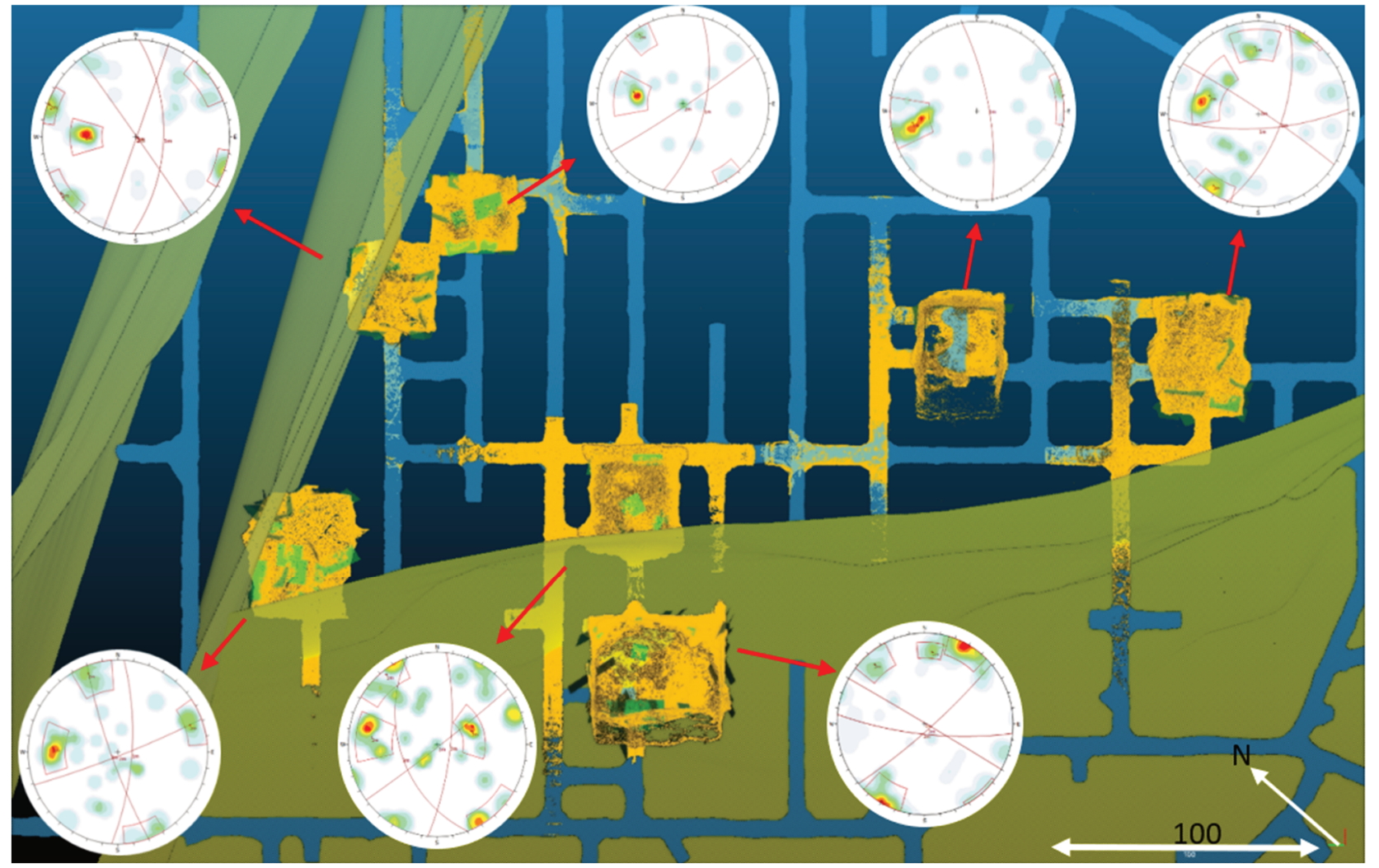

Figure 11 Mining area: stope voids (orange), extracted discontinuities (green), deposit scale structures (yellow) and discontinuities picked stereonets

\section{$6 \quad$ Future work}

The recent introduction of Hovermap at OD has already delivered benefits. The next steps include the transition from research project to standard practice, incorporating the new data acquisition and its analysis into OD's stope closeout workflows. The use of LiDAR technology and analysis can expand into other aspects of mining operations. Software development relating to point cloud analysis in general and the automated identification of discontinuities in particular, will facilitate the making of more informed decisions. Data sets are useful only if interpreted in a timely manner. At OD, there is currently a backlog of 250 to 300 partial to final stope scans that need to be examined to gain more understanding of rock mass characteristics. This characterisation can then be applied to future stoping blocks to make more informed decisions, thus helping avoid issues such as overbreak and underbreak. Of course, with further use of this system and programs, this process will be updated accordingly, improving safety, efficiency and ensuring better decisions are made.

\section{Conclusion}

The development of the semi-autonomous, GPS unassisted hover map technology mounted on a drone has allowed access to previously inaccessible structural information. This information, once extracted from the scans, supplements existing data and is expected to improve data coverage. This will lead to a better understanding of the structure of the rock mass and its consequent failure mechanisms. This technology and 
methodology are currently accessible and can easily be implemented on any site. Overall, this will allow optimisation and improvement of current and future processes. As the understanding of rock mass characteristics increases, this should lead to an improvement of mining processes and an increase in safety.

\section{References}

BHP 2020, Annual Report 2020, https://www.bhp.com/investor-centre/annual-report-2020/annual-report-2020/

Cloudcompare 2020, Cloudcompare, version 2.10.2, computer software, Telecom ParisTech, Paris, http://www.cloudcompare.org/ Deswik 2019, Deswik.CAD, version 2018.3.1188, computer software, Deswik, Brisbane, https://www.deswik.com/productdetail/deswik-cad/

Jones, E, Reardon, D \& Hrabar, S 2019, 'The use of automated drones in underground hard rock mines', in W Joughin (ed.), Proceedings of the Ninth International Conference on Deep and High Stress Mining, The Southern African Institute of Mining and Metallurgy, Johannesburg, pp. 323-334, https://doi.org/10.36487/ACG_rep/1952_24_Jones

Jones, E, Sofonia, J, Canales, C, Hrabar, S \& Kendoul, F 2020, 'Applications for the Hovermap autonomous drone system in underground mining operations', The Journal of the Southern African Institute of Mining and Metallurgy, vol. 120, pp. 1-8.

Roscience 2020, Dips, version 8.002, computer software, Rocscience, Toronto, https://www.rocscience.com/

Vallejos, CAC 2019, Structural recognition and rock mass characterisation in underground mines: A UAV And LiDAR Mapping Based Approach, MSc thesis, Universidad De Concepción, Concepción.

Woolmer, DJ, Jones, E, Taylor, J, Baylis, C \& Kewe, D 2021, 'Use of Drone based Lidar technology at Olympic Dam Mine and initial Technical Applications', Proceedings of the Underground Operators Conference 2021, Australasian Institute of Mining and Metallurgy, Melbourne, submitted for publication. 